\title{
GERAÇÃO DE RESÍDUOS MADEIREIROS DO SETOR DE BASE FLORESTAL NA REGIÃO METROPOLITANA DE BELÉM, PARÁ
}

\section{GENERATION OF WOOD WASTE FROM THE FOREST BASED SECTOR IN THE METROPOLI- TAN REGION OF BELÉM, PARÁ STATE}

\author{
Wilson Fernandes Ramos ${ }^{1}$ Maria de Lourdes Pinheiro Ruivo ${ }^{2}$ Mário Augusto Gonçalves Jardim ${ }^{3}$ \\ Larissa Melo de Sousa ${ }^{4}$
}

\section{RESUMO}

A indústria de base florestal é relevante para a economia mundial, pois seus produtos primários e secundários são usados como matéria-prima para outros setores e serviços gerando emprego e renda para a população e tributos para o Estado. No Brasil, a indústria florestal se destaca pela riqueza em madeira, porém, gera elevada quantidade de resíduos. O objetivo do trabalho foi avaliar a quantidade e os fatores responsáveis pela produção de resíduos madeireiros das indústrias de base florestal da Região Metropolitana de Belém, Pará. Para tanto, foram consideradas um universo de 120 empresas que realizavam o processamento primário e secundário, bem como movelarias. Os dados foram coletados por meio de entrevistas semiestruturadas aplicadas em uma amostra de 31 empresas e observações in loco. As variáveis foram analisadas através da estatística descritiva e correlação de Pearson. Verificou-se que as empresas geraram um total de $12,3 \mathrm{mil} \mathrm{m}^{3} /$ mês, com média de $398,9 \mathrm{~m}^{3} /$ mês de resíduo. O número de espécies madeireiras influenciou na quantidade de resíduos. Conclui-se, que a partir da comparação com outras pesquisas com esse mesmo tema, que as empresas geram quantidade significativa de resíduo em consequência do número de espécies processadas.

Palavras-chave: produção; economia florestal; ambiente.

\begin{abstract}
The forest based industry is relevant to the world's economy, since its primary and secondary products are used as raw material for other sectors and services generating employment and income for the population and taxes for the state. In Brazil, the forest industry stands out for the wealth of wood, but it generates a high amount of waste. The objective of this study was to evaluate the quantity and the factors responsible for the production of wood residues from the forest-based industries of the metropolitan region of Belém, Pará state. For this purpose, a total of 120 companies were considered that carried out primary and secondary processing, as well as furniture. The data were collected through semi-structured interviews applied in a sample of 31 companies and observations in loco. The variables were analyzed through descriptive statistics and Pearson correlation. It was verified that the companies generated a total of 12.3 thousand $\mathrm{m}^{3} / \mathrm{month}$, with a mean of $398.9 \mathrm{~m}^{3} /$ month of waste. The number of timber species influenced the amount of waste. It can be concluded from the comparison with other researches with this same theme that the companies generate a significant amount of waste as a consequence of the number of species processed.
\end{abstract}

Keywords: production; Forest economics; environment.

1 Agrônomo, Doutorando do Programa de Pós-graduação em Ciências Ambientais (UFPA/MPEG/Embrapa), Rua Augusto Corrêa, 1, Guamá, CEP 66075-110. Belém (PA), Brasil. wilson.f.ramos18@hotmail.com

2 Geóloga, Dr ${ }^{a}$., Pesquisadora do Museu Paraense Emílio Goeldi, Coordenação de Ciências da Terra e Ecologia (COCTE), Av. Presidente Tancredo Neves, 1901, Terra Firme, CEP 66077-830, Belém (PA), Brasil. ruivo@museu-goeldi.br

3 Engenheiro Florestal, Dr., Pesquisador do Museu Paraense Emílio Goeldi, Coordenação de Botânica (COBOT), Av. Presidente Tancredo Neves, 1901, Terra Firme, CEP 66077-830, Belém (PA), Brasil. jardim@museu-goeldi.br

4 Engenheira Florestal, MSc., Doutoranda do Programa de Pós-graduação em Ciências Florestais, Universidade Federal da Amazônia, Av. Presidente Tancredo Neves, 2501, Terra Firme, CEP 66.077-830, Belém (PA), Brasil. larissa_melo_sousa@hotmail.com

Recebido para publicação em 8/04/2017 e aceito em 20/11/2017

Ci. Fl., v. 28, n. 4, out. - dez., 2018 


\section{INTRODUÇÃO}

A indústria de base florestal é importante para a economia mundial, pois seus produtos primários e secundários são usados como matéria-prima para outros setores econômicos como da construção civil e do automobilismo. Essa indústria possui destaque na economia brasileira em virtude da geração de renda, tributos, divisas, empregos e, recentemente, a preservação ambiental (NUNES; MELO; TEIXEIRA, 2012), principalmente com o reflorestamento de áreas abandonadas para diminuir a pressão na floresta primária.

Os principais produtos obtidos por essa indústria são postes, madeira serrada, lâminas de madeira, painéis colados, compensados, aglomerados, chapas duras de fibra, chapas de fibras de média densidade, celulose e papel, além de energia e móveis. Ademais, essas indústrias são responsáveis, em alguns casos, pelo plantio e extração da madeira (SOCIEDADE BRASILEIRA DE SILVICULTURA, 2007).

No Brasil, a indústria florestal apresenta grande riqueza de matéria-prima madeireira oriunda da floresta Amazônia perfazendo em torno de 90\% da produção florestal (CASTRO; SILVA, 2007). Ademais, o setor madeireiro apresenta grande importância na economia da Região Amazônica (LIMA; SILVA, 2016). Entretanto, essa vantagem se contrapõe aos impactos ambientais que esta atividade juntamente com mineração e a agropecuária trouxeram para a Região (MELLO; ARTAXO, 2017).

A economia da indústria madeireira é notória e pode ser avaliada em termos de postos de trabalho e número de indústrias na Região. Para o SFB e IMAZON (SERVIÇO FLORESTAL BRASILEIRO; INSTITUTO DO HOMEM E MEIO AMBIENTE DA AMAZÔNIA, 2010) em 2009, o setor na Amazônia legal gerou cerca de 204 mil postos de trabalho com aproximadamente duas mil indústrias localizadas em 71 polos madeireiros, e destas mil indústrias no estado do Pará.

Um dos grandes polos madeireiros do Estado do Pará é a Região Metropolitana de Belém, cujo consumo de madeira em tora foi superior a $697 \mathrm{mil} \mathrm{m} /$ ano gerando 13 mil empregos diretos e indiretos em 2009 distribuídos em 31 empresas e contratando em torno de 2.540 empregados (RAMOS; RUIVO; SOUSA, 2016). No Pará, destacam-se ainda os polos de Paragominas, Breves e Tomé-Açú (SERVIÇO FLORESTAL BRASILEIRO; INSTITUTO DO HOMEM E MEIO AMBIENTE DA AMAZÔNIA, 2010).

Historicamente, no Pará, até o fim da década de 50, a exploração madeireira era realizada de forma esporádica e se concentrava em florestas de várzea as margens dos rios da Região. Porém, houve uma intensificação da exploração madeireira a partir dos anos 80, com a melhoria da infraestrutura rodoviária, a qual permitiu o acesso às extensas áreas de reservas florestais (VERÍSSIMO et al., 2006). A combinação desses aspectos com a exaustão das florestas da Ásia e do sul e sudeste do Brasil, tornou a exploração madeireira na Amazônia uma atividade de grande relevância socioeconômica para a região (LIMA; SILVA, 2005). Porém, essa atividade implantou um modelo de grande crescimento econômico seguido por rápido colapso que ocasionou problemas socioambientais devido à degradação da floresta (LENTINI et al., 2005).

Outro problema em relação ao setor de base florestal é a questão dos resíduos gerados pelas indústrias. No Brasil, estima-se que sejam gerados cerca de 30 milhões de toneladas de resíduos de madeira anualmente e 91 \% são gerados nas indústrias madeireiras (TUOTO, 2009). A maioria dos resíduos do setor é gerada no processamento primário da madeira (FINOTTI et al., 2006).

Na Região Amazônica, estima-se que sejam gerados mais de 8 milhões de metros cúbicos de resíduo e no Pará cerca de 4 milhões de $\mathrm{m}^{3}$ de resíduos (SERVIÇO FLORESTAL BRASILEIRO; INSTITUTO DO HOMEM E MEIO AMBIENTE DA AMAZÔNIA, 2010), além de 59\% das perdas do processamento da madeira nativa, o que caracteriza um cenário de baixa tecnologia associado a um mau aproveitamento da madeira (BATISTA et al., 2015).

$\mathrm{Na}$ Região Metropolitana de Belém estima-se que essas perdas alcancem patamares de $48 \% \mathrm{em}$ serrarias da região. O resíduo é gerenciado de maneira incorreta, pois é depositado no pátio das empresas ou em vicinais nas suas proximidades. Este desperdício impossibilita a geração de renda pela empresa (RAMOS; RUIVO; SOUSA, 2016). Na região sul, os rendimentos médios líquidos encontrados foram de 56,58\%, 43,5\% e 39,2\% (BARBOSA et al., 2014; CUNHA et al., 2015). O objetivo do trabalho foi avaliar a quantidade e os fatores responsáveis pela produção de resíduos madeireiros das indústrias de base florestal da Região Metropolitana de Belém. 


\section{MATERIAL E MÉTODO}

O estudo foi realizado na Região Metropolitana de Belém (RMB) (Figura 1) criada em 1973 e constituída atualmente pelos municípios de Ananindeua, Belém, Benevides, Marituba, Santa Barbara do Pará e Santa Isabel do Pará. A área de estudo encontra-se em um clima classificado como Af, segundo Köppen, sendo um clima quente e úmido, com temperatura média anual de $26^{\circ} \mathrm{C}$, com umidade relativa do ar girando em torno de $90 \%$, o índice pluviométrico está entre $2.300 \mathrm{~mm}$ a $3.000 \mathrm{~mm}$ por ano e apresenta uma Latitude: $01^{\circ} 23^{\prime} .6$ Sul e Longitude: $048^{\circ} 29^{\prime} .5$ Oeste.

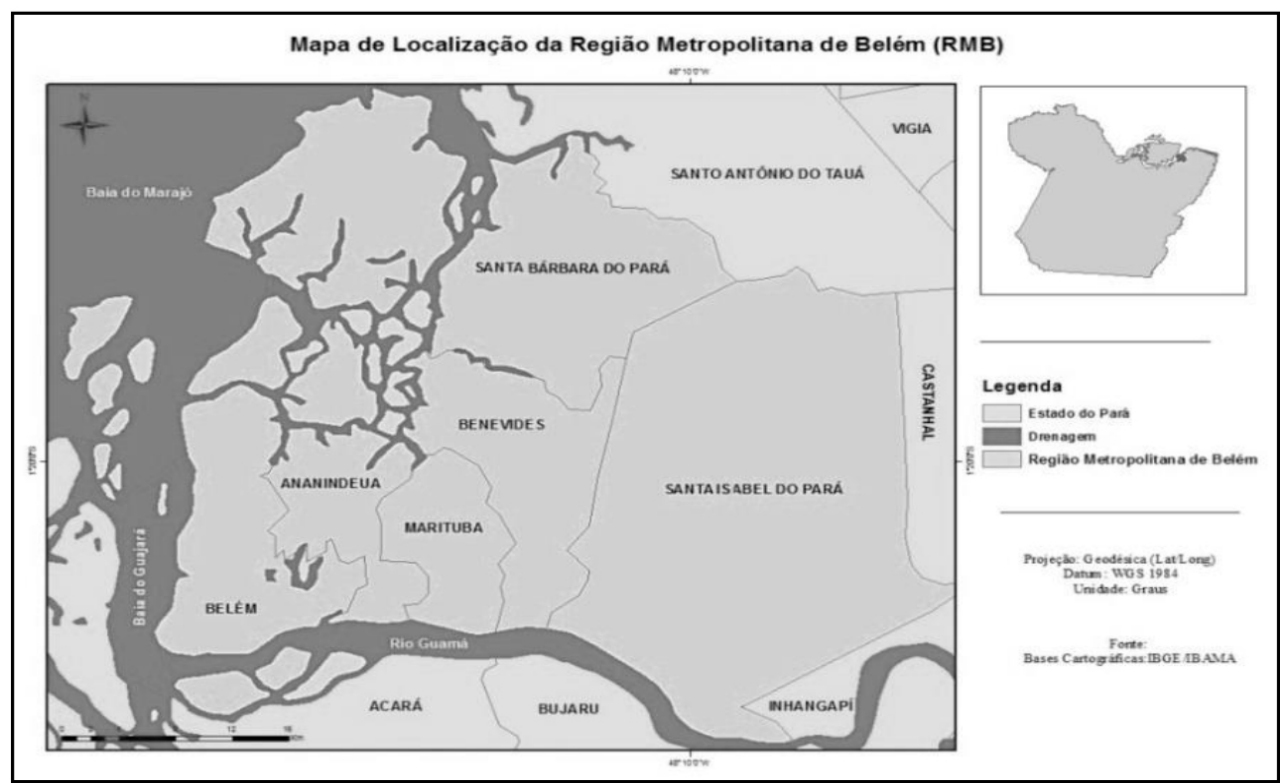

FIGURA 1: Mapa da Região Metropolitana de Belém. Fonte: Base cartográfica IBGE (2010).

FIGURE 1: Map of the Metropolitan Region of Belém. Source: IBGE Cartographic Base (2010).

A Região metropolitana de Belém (RMB) foi escolhida como local desta pesquisa pelo fato de ser um dos grandes polos madeireiros do Estado do Pará e concentrar um grande número de empresas desse setor. Os dados sobre o número de empresas do setor foram obtidos junto à Secretária Estadual do Meio Ambiente Sustentabilidade (SEMAS) e à Associação das Indústrias Exportadoras de Madeira do Estado do Pará (AIMEX). Foram consideradas 120 empresas cujo processamento primário, secundário ou terciário abrange a serraria com desdobro de madeira, a serraria sem desdobro de madeira e movelarias. Em seguida foi aplicada a técnica de amostragem não probabilística por conveniência onde foram selecionadas $31 \mathrm{em}$ presas localizadas na Região Metropolitana de Belém no período de maio a julho de 2015. Os dados foram coletados através da entrevista semiestruturada com perguntas abertas e fechadas (SEVERINO, 2007) aos proprietários (titular do empreendimento na forma da lei); representante legal (mandatário legalmente constituído através junto a SECTAM-PA); responsável técnico (Engenheiro Florestal devidamente habilitado pelo Conselho Regional de Engenharia, Arquitetura e Agronomia - CREA-PA). A escolha desses atores, para a realização da pesquisa foi por meio do critério de conhecimento técnico da atividade e do poder de decisão na empresa. O Formulário com as perguntas encontra-se no Apêndice 1. Os dados foram analisados através da estatística descritiva e Correlação de Pearson com auxílio do Programa Excel.

\section{RESULTADOS}

As empresas apresentaram variação na quantidade de resíduos produzidos que foi influenciada pelo consumo de matéria-prima (madeira) e produção. Dessa forma, considerando todas as empresas visitadas na Região Metropolitana de Belém (31), a média foi de 398,9 m³/mês de resíduo. Sendo o mínimo de 
$0,63 \mathrm{~m}^{3}$ de resíduo mensal e o máximo de $919 \mathrm{~m}^{3}$ por mês de resíduo gerado. A soma do resíduo madeireiro gerado por todas as empresas foi de $12,3 \mathrm{mil} \mathrm{m} / \mathrm{mês}$. A quantidade de resíduo gerado mensalmente por cada município é bem distinta, devido ao tamanho dos empreendimentos serem diferentes e principalmente o número de empresas de cada município.

Verificou-se que os municípios de Belém, Benevides e Ananindeua geraram juntos mais de $80 \%$ de todo o resíduo da Região metropolitana, com 4,20 mil (34,03\%), 3,65 mil (29,54\%) e 2,17 mil m³ $/ \mathrm{mês}$ $(17,59 \%)$, respectivamente. Já Santa Bárbara do Pará, Marituba e Santa Isabel do Pará geram menos que $20 \%$ desse montante, com valores de 1,4 mil $(12 \%), 840(6,8 \%)$ e $0,63 \mathrm{~m}^{3}(0,01 \%)$ de resíduos mensais respectivamente (Figura 2).

Porém, o município de Belém, Ananindeua e Benevides apresentam a maior quantidade de resíduos gerados por mês devido ao maior número de empresas visitadas. Os resultados apontam que Benevides foi o município que teve a maior média de geração de resíduos com $521,9 \mathrm{~m}^{3} / \mathrm{mês}$, seguido por Belém com 382,5 $\mathrm{m}^{3}$ e Ananindeua com $310,8 \mathrm{~m}^{3}$ mensais (Tabela 1). A correlação entre a quantidade de resíduo madeireiro gerado mensalmente e o número de espécies utilizadas no processamento da madeira mostra correlação positiva considerada moderada, com o de $r=0,56$ (Figura 3).

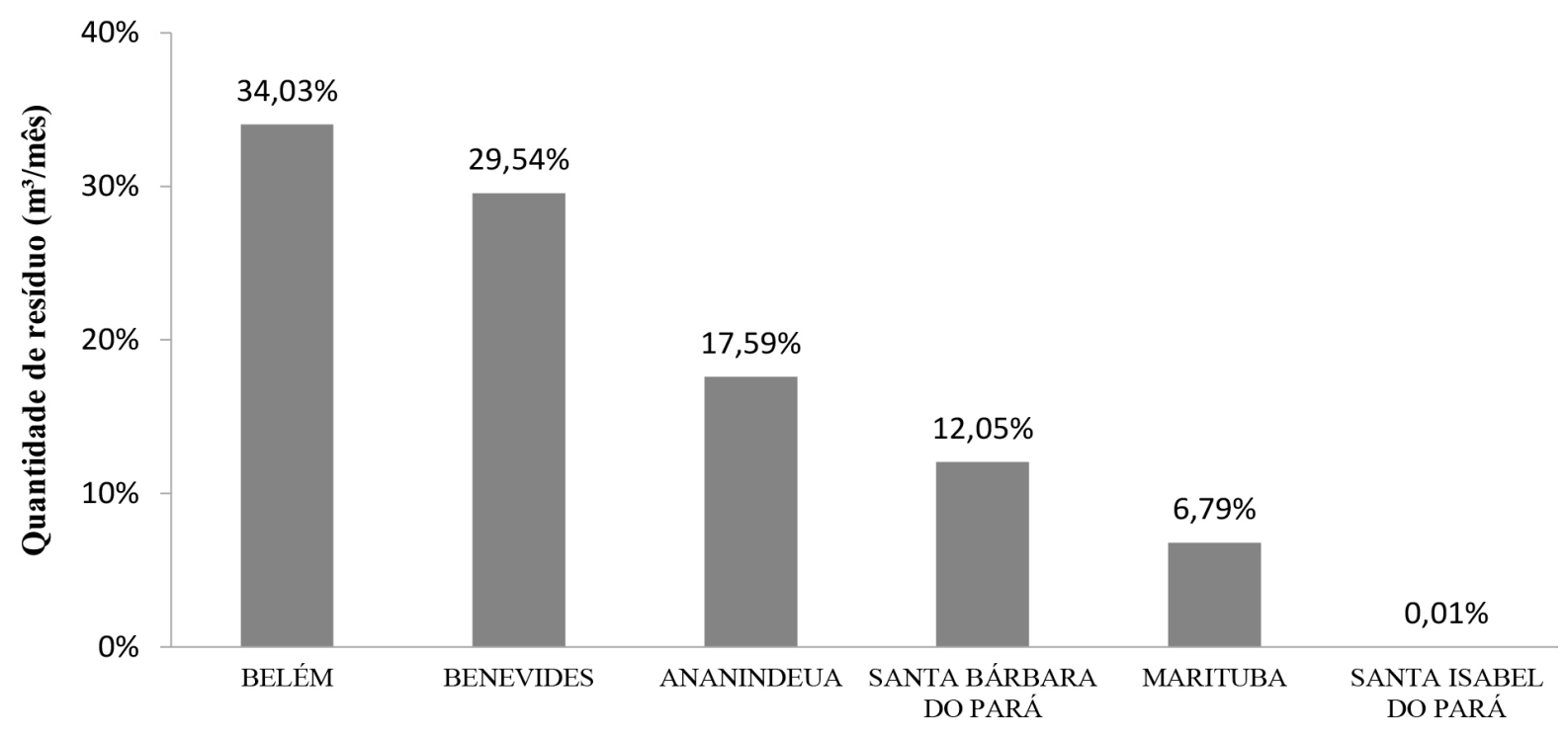

FIGURA 2: Montante de resíduo madeireiro (\%) gerado em cada município da Região Metropolitana de Belém. FIGURE 2: Amount of timber residue (\%) generated in each municipality of the Metropolitan Region of Belém. 
TABELA 1: Valor da média, mínimo e máximo e quantidade mensal de resíduos gerados pelos municípios de Ananindeua, Belém e Benevides ( $\left.\mathrm{m}^{3} / \mathrm{mês}\right)$.

TABLE 1: Value of the average, minimum and maximum and monthly amount of waste generated by the municipalities of Ananindeua, Belém and Benevides $\left(\mathrm{m}^{3} /\right.$ month).

\begin{tabular}{lccccccc}
\hline \multirow{2}{*}{ Tipos del Uso } & \multicolumn{2}{c}{1998} & \multicolumn{2}{c}{2008} & \multicolumn{2}{c}{ Cambio } & Tasa de Cambio \\
\cline { 2 - 7 } & ha & $\%$ & ha & $\%$ & ha & $\%$ & Anual \\
\hline 1 Áreas urbanas e Industriales & 25,803 & 0,7 & 35,686 & 1,0 & 9,882 & 38,3 & 3,2 \\
2 Terrenos agrícolas & 965,145 & 26,0 & 829,508 & 22,4 & $-135,637$ & $-14,1$ & $-1,5$ \\
3 Praderas y matorrales & 637,163 & 17,2 & 498,496 & 13,5 & $-138,667$ & $-21,8$ & $-2,5$ \\
4.1 Plantaciones & 958,697 & 25,9 & 1.227 .788 & 33,1 & 269,091 & 28,1 & 2,5 \\
4.2 Bosque nativo & 776,436 & 21,0 & 768,552 & 20,7 & $-7,883$ & $-1,0$ & $-0,1$ \\
4.3 Bosque mixto & 53,294 & 1,4 & 56,642 & 1,5 & 3,348 & 6,3 & 0,6 \\
5 Humedales & 12,027 & 0,3 & 11,595 & 0,3 & -432 & $-3,6$ & $-0,4$ \\
6 Áreas desprovistas de & 133,976 & 3,6 & 132,518 & 3,6 & $-1,458$ & $-1,1$ & $-0,1$ \\
vegetación & 90,692 & 2,4 & 90,468 & 2,4 & -224 & $-0,2$ & 0,0 \\
7 Nieves y glaciares & 52,558 & 1,4 & 54,742 & 1,5 & 2,184 & 4,2 & 0,4 \\
8 Cuerpos de agua & 211 & 0,0 & 8 & 0,0 & -203 & $-96,1$ & $-32,6$ \\
9 Áreas no reconocidas & 3.706 .003 & \multicolumn{7}{c}{3.706 .003} & & & \\
\hline Total & & \multicolumn{7}{c}{}
\end{tabular}

Quanto maior a quantidade de espécies processadas por empresa maior a geração de resíduos. O $\mathrm{R}^{2}$ mostra o coeficiente de determinação, com valor de 0,3237 , ou seja, $32 \%$ da variável número de espécies processadas por empresas explica a quantidade de resíduos por elas gerados, dessa forma $68 \%$ restante são explicadas por outras variáveis como matéria-prima de baixa qualidade, pouca instrução dos operadores, armazenamento incorreto das toras. As variáveis da quantidade de resíduo madeireiro gerado mensalmente e idade média do maquinário foram correlacionadas.

Os resultados apontam que a correlação de Pearson foi de 0,015 , sendo considerada uma correlação linear não significativa (Figura 4) ou seja, a idade do maquinário não influencia a geração de resíduos. $\mathrm{O}^{2}$ mostra o coeficiente de determinação, com valor de 0,0002 , ou seja, $0,02 \%$ da variável idade do maquinário explica a quantidade de resíduos por elas gerados, dessa forma quase $100 \%$ é explicada por outras variáveis.

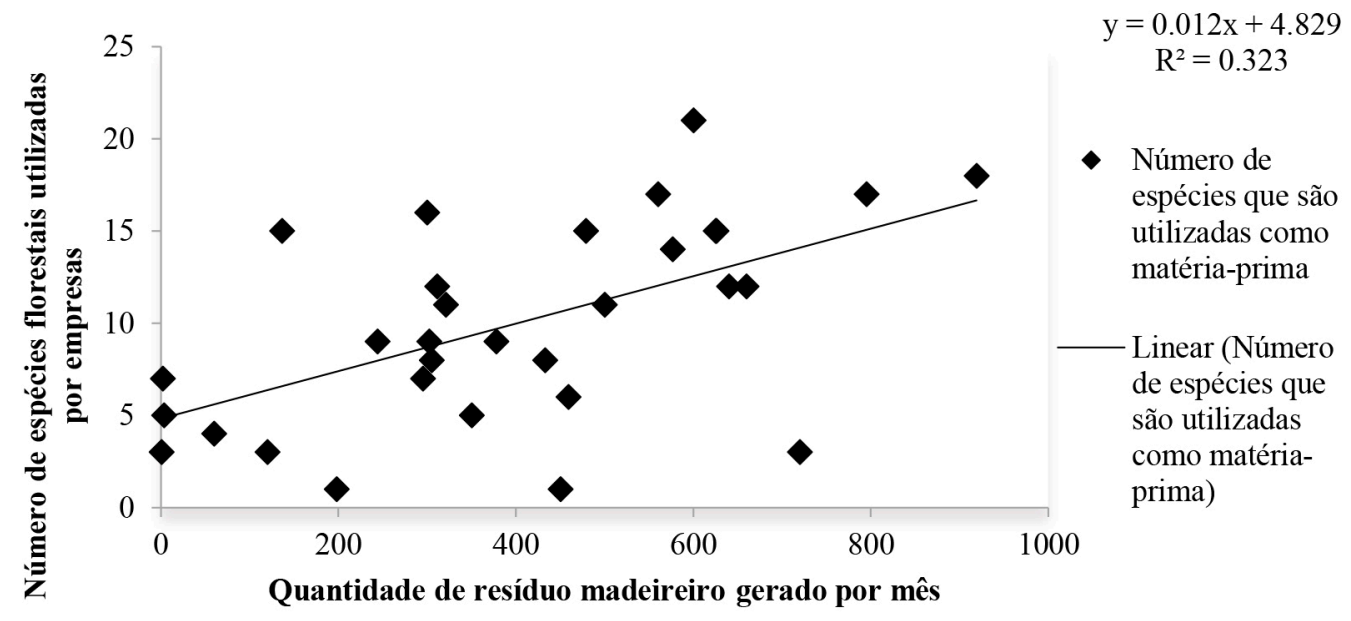

FIGURA 3: Correlação entre quantidade de resíduo e número de espécies processadas.

FIGURE 3: Correlation between amount of residue and number of species processed. 


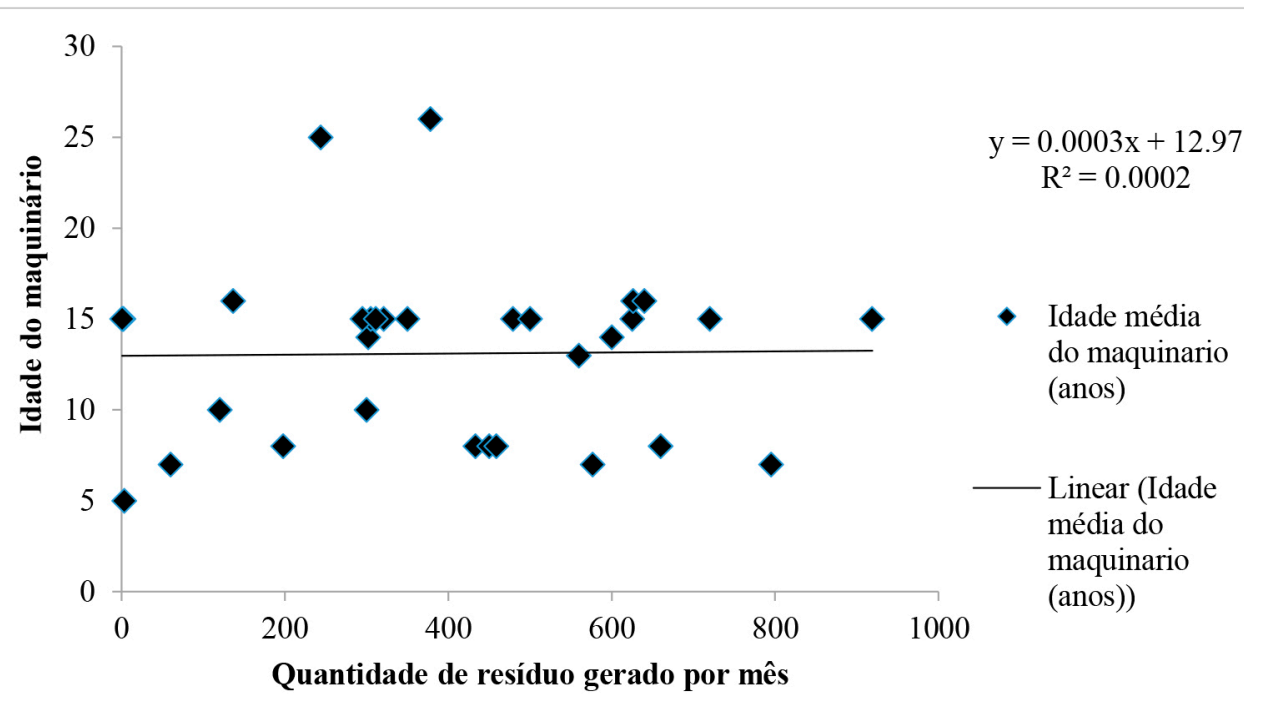

FIGURA 4: Correlação entre quantidade de resíduo e a idade média do maquinário.

FIGURE 4: Correlation between quantity of waste and the average age of the machinery.

\section{DISCUSSÃO}

Estima-se que no Brasil sejam gerados 30 milhões de toneladas de resíduos de madeira anualmente, destes 91\% são gerados nas indústrias madeireiras (TUOTO, 2009). Na Amazônia legal, em 2009, foi estimada a geração de 8,4 milhões de $\mathrm{m}^{3}$ de resíduos do processamento da madeira (SERVIÇO FLORESTAL BRASILEIRO; INSTITUTO DO HOMEM E MEIO AMBIENTE DA AMAZÔNIA, 2010).

Esses valores expressam baixa eficiência das empresas do município de Benevides. Isso é decorrente da grande maioria das empresas que atuam neste município realizarem a atividade de processamento primário (serraria com desdobro) que, de acordo com Finotti et al. (2006), é a etapa de processamento que mais gera resíduo.

Essa quantidade de resíduo gerado destaca o baixo rendimento operacional de $52 \%$ encontrado em serrarias (processamento primário) da Região Metropolitana de Belém, excluindo o município de Santa Isabel do Pará. Porém, quando se consideram as indústrias de processamento secundário esse rendimento aumenta, chegando a aproximadamente 60\% (RAMOS; RUIVO; SOUSA, 2016).

Um fator que pode contribuir para a grande geração de resíduo é a facilidade de obtenção da matéria-prima madeira, a qual é abundante em determinadas regiões (DONATO; TAKENAKA, 2016). Por essas razões, os rendimentos obtidos por serrarias no desdobro da madeira variam de uma indústria para outra, sendo o diagnóstico fundamental para estabelecer as possibilidades de aproveitamento. Para Brand et al. (2002), a quantidade de resíduo é um dos fatores que determinam a potencialidade de seu uso e deve ser analisada em termos de sua geração e utilização.

Ressalta-se que, de acordo com Ramos, Ruivo e Sousa (2016), são processadas aproximadamente 27 espécies florestais em empresas na RMB, número bem maior do que é utilizado na região sul e sudeste, as quais utilizam em torno de 3 a 5 espécies em seu processamento (LIMA; SILVA, 2005). Esse alto número de espécie dificulta o rearranjo dos maquinários o que diminui a eficiência, pois, cada espécie apresenta características de crescimento diferentes e apresentam diâmetros que também diferem.

A idade do maquinário é um fator importante na eficiência das indústrias de base florestal e que a idade avançada diminui o rendimento operacional das empresas (BATISTA et al., 2015). Nesta pesquisa, os resultados não sugerem que a idade do maquinário tenha influenciado na geração de resíduo. Dessa forma, as características externas das toras são determinantes para se obter qualidade e rendimento satisfatórios no processo de beneficiamento da madeira serrada (SILVA, 2011) e a presença de podridões no interior das toras e problemas técnicos nos maquinários levam a um aumento na geração de resíduos (ROCHA, 2007). 
Dentre outros fatores que podem influenciar a geração de resíduos está a distância do local de origem das madeira, a grande heterogeneidade das espécies usadas, principalmente em relação ao diâmentro, as formas incorretas de armazenagem das toras nos pátios das empresas layout da indústria madeireira, características e condições operacionais do maquinário, produtos finais, mão de obra e principalmente e automação da serraria (ROCHA, 2007). Para Batista et al. (2013), o diâmetro da tora é fator importante para a geração de residuos, sendo a classe diamétrica $(29,0-31,9 \mathrm{~cm})$ a que apresenta o melhor rendimento, de $66,6 \%$.

Para diminuir a quantidade de residuos gerados por essas indústrias, investimento em equipamentos mais eficientes como máquinas que possam se adequar ao diâmetro da tora, em mão de obra qualificada e no aproveitamento desses resíduos pode tornar essa atividade menos impactante, diminuindo os problemas causados à população e ao ambiente, e proporcionando maior credibilidade a esse setor (RAMOS et al., 2017).

\section{CONCLUSÃO}

As empresas variaram na quantidade de resíduos produzidos em consequência do tamanho dos empreendimentos e principalmente o número de empresas de cada município. Quanto maior a quantidade de espécies florestais utilizadas no processamento, maior a quantidade de resíduos gerada. Por outro lado, a idade do maquinário não afetou a geração de resíduos. Para uma análise mais abrangente é necessario avaliar outras variáveis atuantes no processo da geraçaõ de resíduos.

\section{REFERÊNCIAS}

BARBOSA, L. C. et al. Avaliação dos resíduos de uma serraria para a produção de celulose kraft. Ciência Florestal, Santa Maria, v. 24, n. 2, p. 491-500, abr./jun. 2014.

BATISTA, D. C. et al. Desdobro de Eucalyptus grandis com motosserra, Parte 1-Análise do desempenho operacional. Ciência Florestal, Santa Maria, v. 23, n. 3, p. 471-481, jul./set. 2013.

BATISTA, D. C. et al. Desempenho operacional de uma serraria de pequeno porte do Município de alegre, Espírito Santo, Brasil. Floresta, Curitiba, v. 45, n. 3, p. 487 - 496, jul./set. 2015.

BRAND, M. A. et al. Caracterização do rendimento e quantificação dos resíduos gerados em serraria através do balanço de materiais. Floresta, Curitiba, v. 32, n. 2, p. 247-259, abr./jun. 2002.

CASTRO, E. M. R.; SILVA, R. N. M. Setor madeireiro, dinâmica de atores e política florestal. In: VENTURIERI, A. (Ed.). Zoneamento ecológico-econômico da área de influência da rodovia BR 163 (Cuiabá-Santarém) diagnóstico do meio socioeconômico, jurídico e arqueologia. Belém: Embrapa Amazônia Oriental, 2007. v. 1. p.169-200.

CUNHA, A. B. et al. Determinação do rendimento de matéria-prima de Eucalyptus benthamii Maiden \& Cambage por meio de diferentes métodos de desdobro. Revista Árvore, Viçosa, MG, v. 39, n. 4, p. 733741, out./dez. 2015.

DONATO, C. J; TAKENAKA, E. M. M. O Aproveitamento de resíduos de madeira para o desenvolvimento sustentável. Periódico Eletrônico Fórum Ambiental da Alta Paulista, Tupã, v. 12, n. 4, 2016.

FINOTTI, A. R. et al. Uso energético de resíduos de madeira na cadeia produtiva de madeira /móveis e possibilidades de geração de créditos de carbono. In: PÓLO MOVELEIRO DA SERRA GAÚCHA. Sistemas de gerenciamento ambiental na indústria moveleira. Caxias do Sul: EDUSP, 2006. IBGE. Bases Cartográficas. Brasília: IBGE, 2010. Disponível em: <https://mapas.ibge.gov.br/bases-e-referenciais/bases-cartograficas/mapas-municipais>. Acesso em: 20 fev. 2017.

LENTINI, M. et al. Fatos florestais da Amazônia. Belém: Imazon, 2005. 110 p.

LIMA, E. G.; SILVA, D. A. Resíduos gerados em indústrias de móveis de madeira situadas no pólo moveleiro de Arapongas - PR. Floresta, Curitiba, v. 35, n. 1, p. 105-116, 2005.

LIMA, J. B.; SILVA, J. M. P. da. Dinâmicas econômicas e ordenamentos territoriais dos grandes projetos de mineração no estado do Pará, 2009-2014: o caso de Paragominas. GEOSABERES - Revista de Estudos Geoeducacionais, Fortaleza, v. 6, n. 3, p. 402-416, 2016.

MELLO, N. G. R.; ARTAXO, P. Evolution of the action plan for prevention and control of deforestation in 
the Brazilian Amazon. Revista do Instituto de Estudos Brasileiros, São Paulo, n. 66, p. 108-129, 2017. NUNES, P. A.; MELO, C. O.; TEIXEIRA, D. A participação do setor madeireiro na economia das microrregiões geográficas do Paraná - 2009. Revista Brasileira de Agropecuária Sustentável, Viçosa, MG, v. 2, n. 1, p. 8-20, jul. 2012.

RAMOS, W. F. et al. Análise da indústria madeireira na Amazônia: gestão, uso e armazenamento de resíduos. Revista Brasileira de Ciências Ambientais, São Paulo, n. 43, p. 1-16, jan/mar. 2017.

RAMOS, W. F.; RUIVO, M. L. P.; SOUSA, L. M. Análise do aspecto produtivo das indústrias madeireiras de processamento primário da Região Metropolitana de Belém. Revista enciclopédia biosfera, Goiânia, v. 13 n. 24, p. 39-50, out./dez. 2016.

ROCHA, M. P. Técnicas de serrarias. In: OLIVEIRA, J. T. S.; FIEDLER, N. C.; NOGUEIRA, M. (Org.). Tecnologias aplicadas ao setor madeireiro. Jerônimo Monteiro: Suprema, 2007. p. 209-270.

SERVIÇO FLORESTAL BRASILEIRO; INSTITUTO DO HOMEM E MEIO AMBIENTE DA AMAZÔNIA. A atividade madeireira na Amazônia brasileira: produção, receita e mercados. Belém: [s. n.], 2010.

SEVERINO, A. J. Metodologia do trabalho científico. 23. ed. rev. e atual. São Paulo: Cortez, 2007.

SILVA, J. L. P. Aproveitamento de resíduos da indústria madeireira para geração de energia elétrica o caso da empresa B K Energia Itacoatiara Ltda. no estado do Amazonas. 2011. Monografia (Especialização em Gestão da Indústria Madeireira e Moveleira) - Universidade Federal do Paraná, Departamento de Ciências Florestais, Curitiba, 2011.

SOCIEDADE BRASILEIRA DE SILVICULTURA. Fatos e números do Brasil florestal. São Paulo: [s. n.], 2007. $110 \mathrm{p}$.

TUOTO, M. Levantamento sobre a geração de resíduos provenientes da atividade madeireira e proposição de diretrizes para políticas, normas e condutas técnicas para promover o seu uso adequado. Projeto PNUD BRA 00/20 - Apoio às Políticas Públicas na Área de Gestão e Controle Ambiental. Curitiba: PNUD, 2009.

VERISSIMO, A. et al. Áreas para a produção florestal manejada: detalhamento do macrozoneamento ecológico econômico do estado do Pará, 2006 (Relatório para o governo do estado do Pará). Belém: [s. n.], 2006. 